\title{
One-pot Synthesis of Tetraphene and Construction of Expanded Conjugated Aromatics
}

Jianbo Wang, Jinzhong Yao, Hailong Wang, Hao Chen, Jingcheng Dong, and Hongwei Zhou *, College of Biological, Chemical Sciences and Engineering, Jiaxing University, Jiaxing 314001, People's Rupublic of China

E-mail:wjb4207@mail.ustc.edu.cn, zhouhw@zju.edu.cn.

\section{Table of Contents}

I. Tables of atom coordinates and absolute energies of $\mathbf{3 a}$ and $\mathbf{3 b}$

II. X-rat- crystal structures and analysis data of compound $\mathbf{2 g}$ 
Compound 3a

\begin{tabular}{|c|c|c|c|}
\hline \multirow{2}{*}{$\begin{array}{l}\text { Atomic } \\
\text { Type }\end{array}$} & \multicolumn{3}{|c|}{ Coordinates } \\
\hline & $X$ & $\mathrm{Y}$ & Z \\
\hline $\mathrm{C}$ & -6.586292 & -4.539426 & -1.458865 \\
\hline $\mathrm{C}$ & -5.239194 & -4.212168 & -1.802560 \\
\hline $\mathrm{C}$ & -4.692993 & -3.016750 & -1.391331 \\
\hline $\mathrm{C}$ & -5.457448 & -2.087148 & -0.630227 \\
\hline $\mathrm{C}$ & -6.808720 & -2.423171 & -0.274362 \\
\hline $\mathrm{C}$ & -7.339876 & -3.672194 & -0.711776 \\
\hline $\mathrm{C}$ & -4.920180 & -0.858170 & -0.201170 \\
\hline $\mathrm{C}$ & -5.653577 & 0.052071 & 0.557551 \\
\hline $\mathrm{C}$ & -7.011055 & -0.290637 & 0.907616 \\
\hline $\mathrm{C}$ & -7.549500 & -1.506020 & 0.487575 \\
\hline $\mathrm{C}$ & -5.110613 & 1.332660 & 1.013850 \\
\hline $\mathrm{C}$ & -5.932357 & 2.202296 & 1.786956 \\
\hline $\mathrm{C}$ & -7.282131 & 1.812990 & 2.111207 \\
\hline $\mathrm{C}$ & -7.793250 & 0.629115 & 1.692133 \\
\hline $\mathrm{N}$ & -4.483941 & -5.124946 & -2.583409 \\
\hline $\mathrm{C}$ & -4.269140 & -6.475031 & -2.279244 \\
\hline $\mathrm{C}$ & -3.482750 & -7.059122 & -3.304574 \\
\hline $\mathrm{C}$ & -3.210172 & -6.015618 & -4.270969 \\
\hline $\mathrm{C}$ & -3.839524 & -4.837549 & -3.794313 \\
\hline $\mathrm{C}$ & -2.509555 & -5.982286 & -5.482230 \\
\hline $\mathrm{C}$ & -2.451414 & -4.791326 & -6.199952 \\
\hline $\mathrm{C}$ & -3.093612 & -3.636823 & -5.720419 \\
\hline $\mathrm{C}$ & -3.795702 & -3.642124 & -4.516883 \\
\hline $\mathrm{C}$ & -4.677793 & -7.209432 & -1.162212 \\
\hline $\mathrm{C}$ & -4.307548 & -8.551259 & -1.097669 \\
\hline $\mathrm{C}$ & -3.542096 & -9.149985 & -2.112876 \\
\hline $\mathrm{C}$ & -3.123715 & -8.408780 & -3.214282 \\
\hline $\mathrm{C}$ & -3.796831 & 1.759020 & 0.724486 \\
\hline $\mathrm{C}$ & -3.310589 & 2.976980 & 1.165406 \\
\hline $\mathrm{C}$ & -4.123601 & 3.827010 & 1.941535 \\
\hline $\mathrm{C}$ & -5.418845 & 3.431757 & 2.246221 \\
\hline $\mathrm{N}$ & -3.619632 & 5.072113 & 2.397377 \\
\hline $\mathrm{C}$ & -4.210436 & 6.325296 & 2.188844 \\
\hline $\mathrm{C}$ & -3.398808 & 7.321991 & 2.788121 \\
\hline $\mathrm{C}$ & -2.270095 & 6.641144 & 3.387732 \\
\hline $\mathrm{C}$ & -2.438169 & 5.257528 & 3.126710 \\
\hline $\mathrm{C}$ & -5.375291 & 6.653706 & 1.489827 \\
\hline $\mathrm{C}$ & -5.729672 & 7.999203 & 1.415432 \\
\hline $\mathrm{C}$ & -4.944800 & 8.998366 & 2.016145 \\
\hline
\end{tabular}




\begin{tabular}{|c|c|c|c|}
\hline $\mathrm{C}$ & -3.778465 & 8.666341 & 2.699091 \\
\hline $\mathrm{C}$ & -1.159108 & 7.074773 & 4.120570 \\
\hline $\mathrm{C}$ & -0.243691 & 6.134878 & 4.585028 \\
\hline $\mathrm{C}$ & -0.433836 & 4.765884 & 4.330376 \\
\hline $\mathrm{C}$ & -1.530798 & 4.307428 & 3.603739 \\
\hline $\mathrm{H}$ & -7.004136 & -5.476179 & -1.813112 \\
\hline $\mathrm{H}$ & -3.662260 & -2.780416 & -1.639179 \\
\hline $\mathrm{H}$ & -8.366163 & -3.922726 & -0.455025 \\
\hline $\mathrm{H}$ & -3.895379 & -0.637236 & -0.482123 \\
\hline $\mathrm{H}$ & -8.574114 & -1.750925 & 0.759816 \\
\hline $\mathrm{H}$ & -7.884662 & 2.496014 & 2.704614 \\
\hline $\mathrm{H}$ & -8.812961 & 0.348129 & 1.943616 \\
\hline $\mathrm{H}$ & -2.021265 & -6.877417 & -5.858696 \\
\hline $\mathrm{H}$ & -1.909283 & -4.752645 & -7.140464 \\
\hline $\mathrm{H}$ & -3.045175 & -2.718330 & -6.299143 \\
\hline $\mathrm{H}$ & -4.295883 & -2.748811 & -4.158164 \\
\hline $\mathrm{H}$ & -5.256474 & -6.751583 & -0.366720 \\
\hline $\mathrm{H}$ & -4.615157 & -9.143260 & -0.239905 \\
\hline $\mathrm{H}$ & -3.270578 & -10.198680 & -2.032388 \\
\hline $\mathrm{H}$ & -2.521440 & -8.870121 & -3.992643 \\
\hline $\mathrm{H}$ & -3.141561 & 1.133254 & 0.128371 \\
\hline $\mathrm{H}$ & -2.305458 & 3.292411 & 0.905025 \\
\hline $\mathrm{H}$ & -6.045367 & 4.072996 & 2.859337 \\
\hline $\mathrm{H}$ & -5.981404 & 5.889938 & 1.014136 \\
\hline $\mathrm{H}$ & -6.631833 & 8.278563 & 0.877918 \\
\hline $\mathrm{H}$ & -5.250274 & 10.038138 & 1.941737 \\
\hline $\mathrm{H}$ & -3.166515 & 9.440773 & 3.154297 \\
\hline $\mathrm{H}$ & -1.016888 & 8.132245 & 4.327823 \\
\hline $\mathrm{H}$ & 0.622860 & 6.459601 & 5.153902 \\
\hline $\mathrm{H}$ & 0.286247 & 4.046041 & 4.710031 \\
\hline $\mathrm{H}$ & -1.676286 & 3.247641 & 3.422733 \\
\hline
\end{tabular}

Energy $=-1725.72372566$ A.U. 


\begin{tabular}{|c|c|c|c|}
\hline \multirow{2}{*}{$\begin{array}{l}\text { Atomic } \\
\text { Type }\end{array}$} & \multicolumn{3}{|c|}{ Coordinates } \\
\hline & $\mathrm{X}$ & $\mathrm{Y}$ & Z \\
\hline $\mathrm{C}$ & 3.486787 & 4.090215 & 0.575341 \\
\hline $\mathrm{C}$ & 2.093650 & 4.231840 & 0.271649 \\
\hline $\mathrm{C}$ & 1.323966 & 3.087097 & 0.183372 \\
\hline $\mathrm{C}$ & 1.872731 & 1.790791 & 0.391308 \\
\hline $\mathrm{C}$ & 3.270750 & 1.668899 & 0.699036 \\
\hline $\mathrm{C}$ & 4.051291 & 2.858838 & 0.781095 \\
\hline $\mathrm{C}$ & 1.087781 & 0.624396 & 0.300415 \\
\hline $\mathrm{C}$ & 1.614391 & -0.649087 & 0.507630 \\
\hline $\mathrm{C}$ & 3.018142 & -0.761575 & 0.825135 \\
\hline $\mathrm{C}$ & 3.803497 & 0.387382 & 0.909287 \\
\hline $\mathrm{C}$ & 0.811532 & -1.869923 & 0.424257 \\
\hline $\mathrm{C}$ & 1.427351 & -3.129709 & 0.672665 \\
\hline $\mathrm{C}$ & 2.834330 & -3.188335 & 0.982739 \\
\hline $\mathrm{C}$ & 3.588952 & -2.063820 & 1.053208 \\
\hline $\mathrm{C}$ & 1.506582 & 5.576703 & 0.062629 \\
\hline $\mathrm{C}$ & 0.203843 & 5.881682 & 0.494324 \\
\hline $\mathrm{C}$ & -0.358226 & 7.136287 & 0.287549 \\
\hline $\mathrm{C}$ & 0.374517 & 8.152324 & -0.348224 \\
\hline $\mathrm{C}$ & 1.681721 & 7.863705 & -0.773769 \\
\hline $\mathrm{C}$ & 2.228173 & 6.600329 & -0.576151 \\
\hline $\mathrm{N}$ & -0.189741 & 9.437619 & -0.552033 \\
\hline $\mathrm{C}$ & 0.044541 & 10.135855 & -1.768923 \\
\hline $\mathrm{C}$ & -1.001740 & 10.028041 & 0.456062 \\
\hline $\mathrm{C}$ & 0.319985 & 11.512773 & -1.758049 \\
\hline $\mathrm{C}$ & 0.538098 & 12.195539 & -2.953154 \\
\hline $\mathrm{C}$ & 0.504066 & 11.519357 & -4.174596 \\
\hline $\mathrm{C}$ & 0.238528 & 10.148430 & -4.187750 \\
\hline $\mathrm{C}$ & 0.000865 & 9.460494 & -2.999344 \\
\hline $\mathrm{C}$ & -2.192220 & 10.689984 & 0.115127 \\
\hline $\mathrm{C}$ & -2.979439 & 11.275367 & 1.104991 \\
\hline $\mathrm{C}$ & -2.606361 & 11.197295 & 2.448414 \\
\hline $\mathrm{C}$ & -1.426966 & 10.532500 & 2.791189 \\
\hline $\mathrm{C}$ & -0.623900 & 9.959771 & 1.806781 \\
\hline $\mathrm{C}$ & -0.563536 & -1.871512 & 0.108357 \\
\hline $\mathrm{C}$ & -1.295732 & -3.043396 & 0.047952 \\
\hline $\mathrm{C}$ & -0.696650 & -4.299168 & 0.300651 \\
\hline $\mathrm{C}$ & 0.662794 & -4.310616 & 0.605348 \\
\hline $\mathrm{C}$ & -1.488732 & -5.549676 & 0.246858 \\
\hline $\mathrm{C}$ & -2.530175 & -5.718308 & -0.682181 \\
\hline $\mathrm{C}$ & -3.272995 & -6.892234 & -0.739481 \\
\hline $\mathrm{C}$ & -3.013204 & -7.947146 & 0.151125 \\
\hline $\mathrm{C}$ & -1.979890 & -7.787339 & 1.089640 \\
\hline
\end{tabular}




\begin{tabular}{|c|c|c|c|}
\hline $\mathrm{C}$ & -1.232484 & -6.615941 & 1.126736 \\
\hline $\mathrm{N}$ & -3.775060 & -9.142206 & 0.105598 \\
\hline $\mathrm{C}$ & -3.139004 & -10.399845 & 0.300441 \\
\hline $\mathrm{C}$ & -5.178014 & -9.088062 & -0.123280 \\
\hline $\mathrm{C}$ & -5.796043 & -10.017268 & -0.975678 \\
\hline $\mathrm{C}$ & -7.172506 & -9.969725 & -1.187876 \\
\hline $\mathrm{C}$ & -7.953090 & -8.988547 & -0.572939 \\
\hline $\mathrm{C}$ & -7.339803 & -8.057439 & 0.267970 \\
\hline $\mathrm{C}$ & -5.966884 & -8.108541 & 0.501546 \\
\hline $\mathrm{C}$ & -3.734075 & -11.382065 & 1.108277 \\
\hline $\mathrm{C}$ & -3.111857 & -12.616112 & 1.287822 \\
\hline $\mathrm{C}$ & -1.882482 & -12.886647 & 0.683208 \\
\hline $\mathrm{C}$ & -1.284346 & -11.908456 & -0.114125 \\
\hline $\mathrm{C}$ & -1.907628 & -10.678256 & -0.314384 \\
\hline $\mathrm{H}$ & 4.096285 & 4.983986 & 0.671982 \\
\hline $\mathrm{H}$ & 0.270849 & 3.162402 & -0.076163 \\
\hline $\mathrm{H}$ & 5.108284 & 2.774695 & 1.023069 \\
\hline $\mathrm{H}$ & 0.035397 & 0.749029 & 0.064643 \\
\hline $\mathrm{H}$ & 4.860791 & 0.286935 & 1.147030 \\
\hline $\mathrm{H}$ & 3.279656 & -4.164284 & 1.160347 \\
\hline $\mathrm{H}$ & 4.649054 & -2.123322 & 1.287912 \\
\hline $\mathrm{H}$ & -0.377232 & 5.124888 & 1.013717 \\
\hline $\mathrm{H}$ & -1.368024 & 7.339494 & 0.629206 \\
\hline $\mathrm{H}$ & 2.264988 & 8.634163 & -1.267717 \\
\hline $\mathrm{H}$ & 3.233330 & 6.402540 & -0.938067 \\
\hline $\mathrm{H}$ & 0.361048 & 12.040553 & -0.810480 \\
\hline $\mathrm{H}$ & 0.750020 & 13.261268 & -2.925666 \\
\hline $\mathrm{H}$ & 0.681649 & 12.053598 & -5.103583 \\
\hline $\mathrm{H}$ & 0.200495 & 9.609383 & -5.130835 \\
\hline $\mathrm{H}$ & -0.219471 & 8.397838 & -3.016709 \\
\hline $\mathrm{H}$ & -2.493424 & 10.742133 & -0.926355 \\
\hline $\mathrm{H}$ & -3.897949 & 11.783434 & 0.822805 \\
\hline $\mathrm{H}$ & -3.226121 & 11.648569 & 3.217976 \\
\hline $\mathrm{H}$ & -1.119007 & 10.469885 & 3.831703 \\
\hline $\mathrm{H}$ & 0.298821 & 9.456188 & 2.077326 \\
\hline $\mathrm{H}$ & -1.076439 & -0.935242 & -0.085239 \\
\hline $\mathrm{H}$ & -2.358976 & -2.993938 & -0.168667 \\
\hline $\mathrm{H}$ & 1.167459 & -5.258888 & 0.773155 \\
\hline $\mathrm{H}$ & -2.737254 & -4.931603 & -1.402387 \\
\hline $\mathrm{H}$ & -4.055436 & -7.001687 & -1.483548 \\
\hline $\mathrm{H}$ & -1.774607 & -8.583030 & 1.798498 \\
\hline $\mathrm{H}$ & -0.461045 & -6.509338 & 1.884319 \\
\hline $\mathrm{H}$ & -5.192079 & -10.773068 & -1.467555 \\
\hline $\mathrm{H}$ & -7.633771 & -10.697234 & -1.850732 \\
\hline $\mathrm{H}$ & -9.024723 & -8.950150 & -0.746409 \\
\hline $\mathrm{H}$ & -7.934725 & -7.292557 & 0.760399 \\
\hline
\end{tabular}




\begin{tabular}{llll}
$\mathrm{H}$ & -5.498125 & -7.391854 & 1.168250 \\
$\mathrm{H}$ & -4.683201 & -11.171786 & 1.591008 \\
$\mathrm{H}$ & -3.586636 & -13.364679 & 1.916826 \\
$\mathrm{H}$ & -1.397086 & -13.847130 & 0.831033 \\
$\mathrm{H}$ & -0.331550 & -12.107095 & -0.598031 \\
$\mathrm{H}$ & -1.445921 & -9.926659 & -0.946857 \\
\hline
\end{tabular}

Energy $=-2190.19245412$ A.U. 
Figure S1 X-ray crystal structures of compound $2 \mathrm{~g}$ (Thermal ellipsoids are drawn at $50 \%$ probability level.)

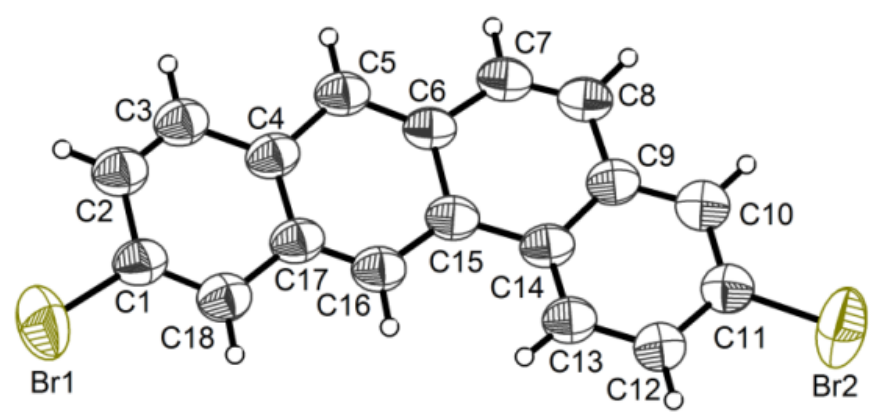

X-ray crystal analysis data for compound $2 \mathbf{g}$ was collected with an Oxford Diffraction Gemini R Ultra diffract-ometer with graphite-monochromated Mo-Ka $(\lambda=0.71073 \AA)$ at $296 \mathrm{~K}$. The structure was solved by direct methods and refined on $\mathrm{F}^{2}$ by full-matrix least-squares methods using the SHELXTL package. A summary of the crystal data and structure refinements of compound $\mathbf{2 g}$ are provided in Table S1.

Table S1 Crystal data and structure refinements for compound $\mathbf{2 g}$

\begin{tabular}{|l|l|}
\hline Compounds & $\mathbf{2 g}$ \\
\hline Empirical formula & $\mathrm{C}_{18} \mathrm{H}_{10} \mathrm{Br}_{2}$ \\
\hline Formula weight & 386.08 \\
\hline Temperature/K & $296(2)$ \\
\hline Crystal system & orthorhombic \\
\hline Space group & $\mathrm{Pna} 2_{1}$ \\
\hline $\mathrm{a} / \AA$ & $13.3649(19)$ \\
\hline $\mathrm{b} / \AA$ & $28.600(3)$ \\
\hline $\mathrm{c} / \AA$ & $7.4174(11)$ \\
\hline$\alpha /{ }^{\circ}$ & 90 \\
\hline$\beta /{ }^{\circ}$ & 90 \\
\hline$\gamma /{ }^{\circ}$ & 90 \\
\hline Volume $/ \AA^{3}$ & $2835.2(7)$ \\
\hline$Z$ & 8 \\
\hline$\rho_{\text {calc }} \mathrm{g} / \mathrm{cm}^{3}$ & 1.809 \\
\hline & \\
\hline
\end{tabular}




\begin{tabular}{|l|l|}
\hline$\mu / \mathrm{mm}^{-1}$ & 5.704 \\
\hline $\mathrm{F}(000)$ & 1504 \\
\hline Radiation & $\mathrm{MoK} \alpha$ \\
\hline Reflections collected & 12326 \\
\hline Independent reflections & 4509 \\
\hline $\mathrm{R}_{\text {int }}$ & 0.0613 \\
\hline Goodness-of-fit on $\mathrm{F}^{2}$ & 1.034 \\
\hline $\mathrm{R}_{1}, w \mathrm{R}_{2}[\mathrm{I}>=2 \sigma(\mathrm{I})]$ & $0.1149,0.2679$ \\
\hline $\mathrm{R}_{1}, w \mathrm{R}_{2}[$ all data $]$ & $0.2003,0.3118$ \\
\hline${ }^{\mathrm{a}} R_{1}=\Sigma|| \mathrm{F}_{\mathrm{o}}|-| \mathrm{F}_{\mathrm{c}}|/ \Sigma| \mathrm{F}_{\mathrm{o}} \mid,{ }^{\mathrm{b}} w R_{2}=\Sigma\left[w\left(\mathrm{~F}_{\mathrm{o}}{ }^{2}-\mathrm{F}_{\mathrm{c}}{ }^{2}\right)^{2}\right] / \Sigma\left[w\left(\mathrm{~F}_{\mathrm{o}}{ }^{2}\right]^{2}\right]^{1 / 2}$. \\
\hline
\end{tabular}




\section{Copies of ${ }^{1} \mathrm{H}$ and ${ }^{13} \mathrm{C}$ NMR Spectra of New Compounds}

${ }^{1} \mathrm{H}$ NMR (400 MHz) spectra of $\mathbf{2 a}$

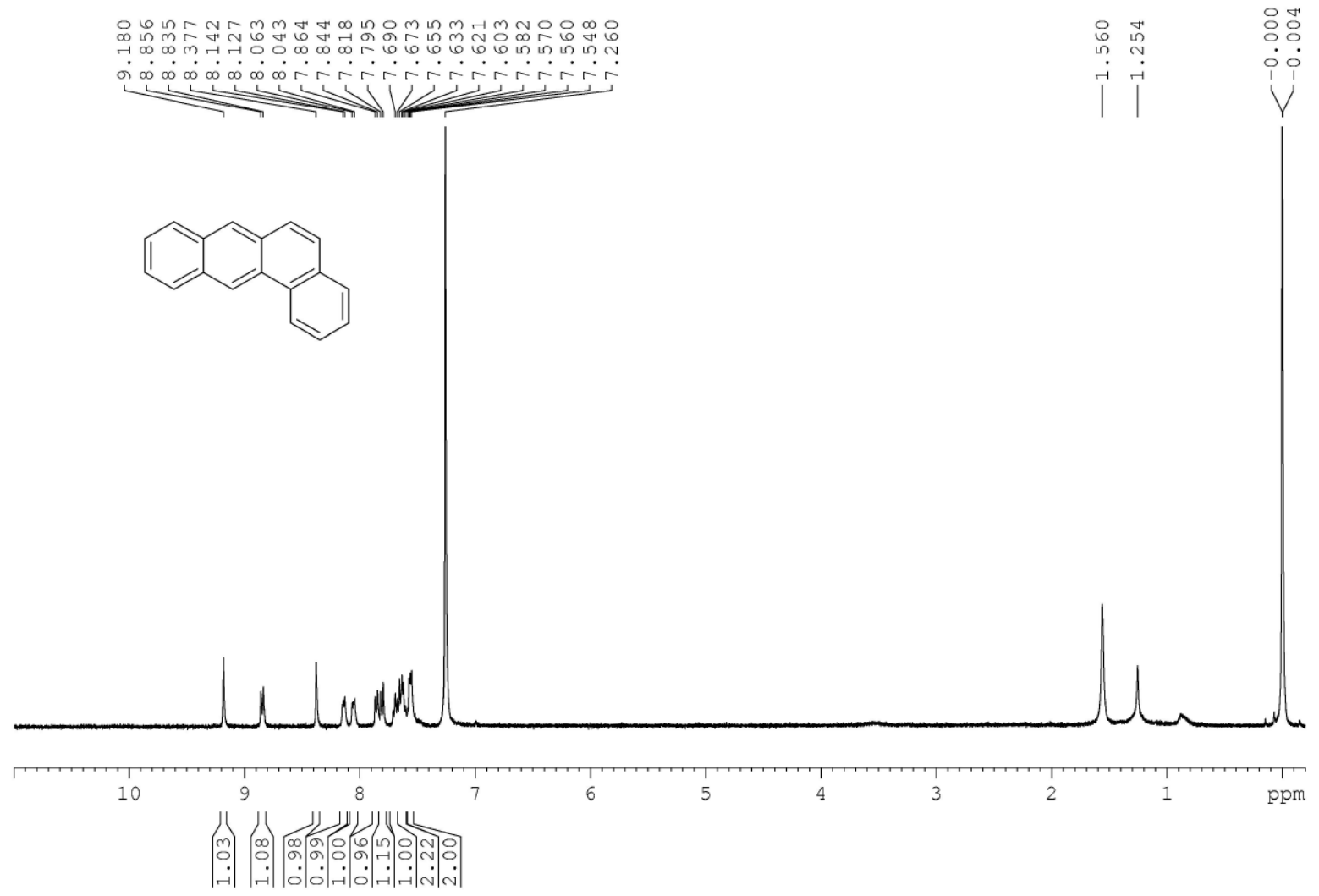

${ }^{13} \mathrm{C}$ NMR (100 MHz) spectra of 2a
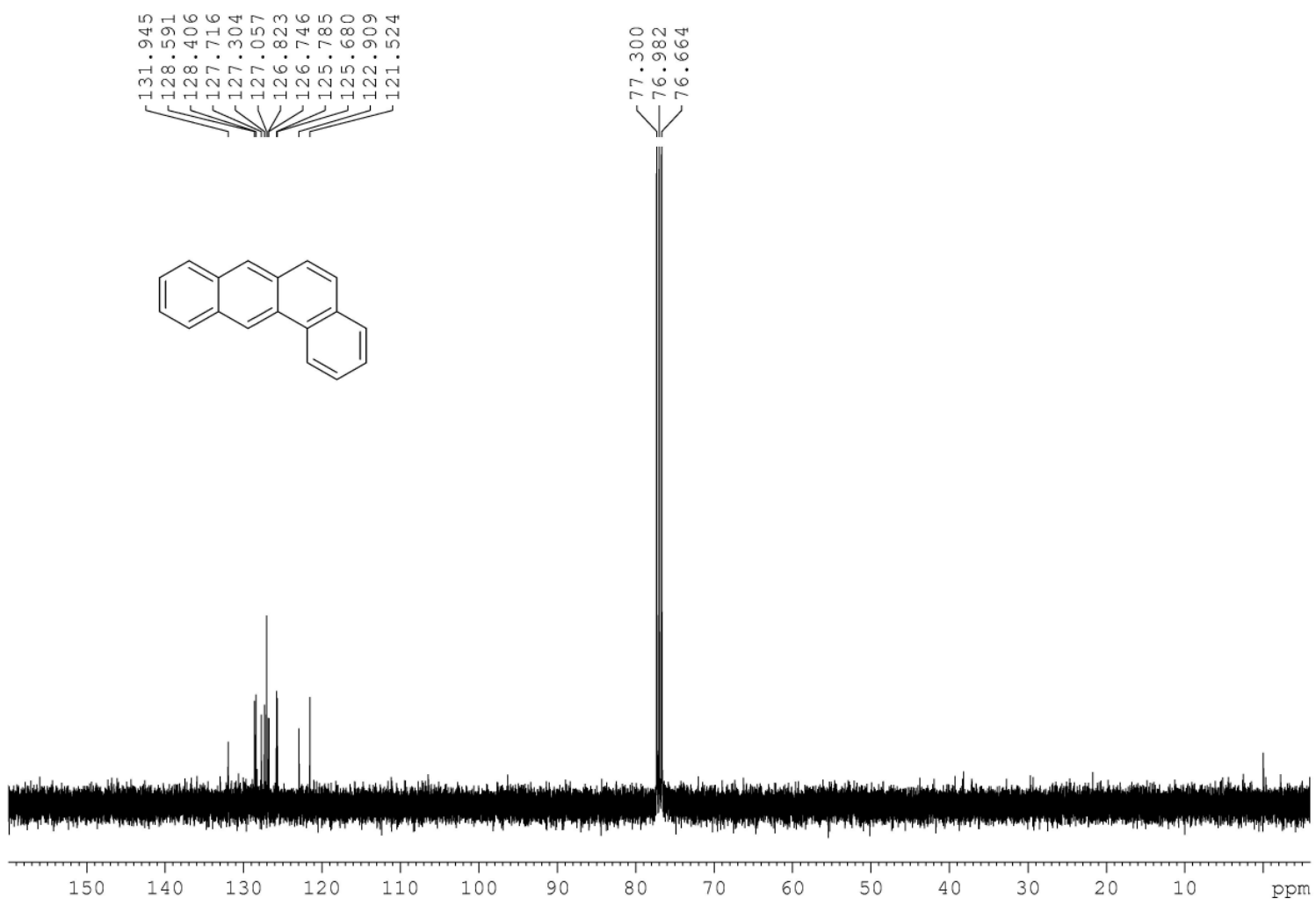
${ }^{1} \mathrm{H}$ NMR (400 MHz) spectra of $\mathbf{2 b}$
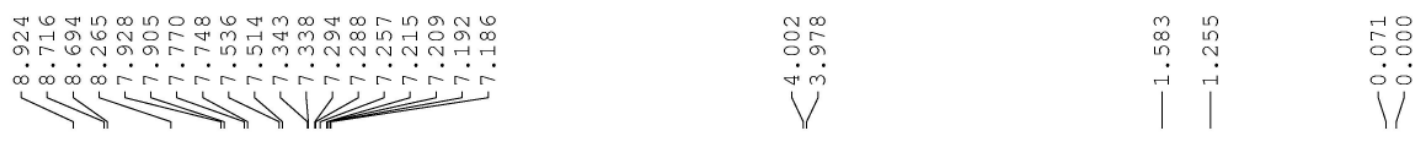<smiles>COc1ccc2cc3ccc4cc(OC)ccc4c3cc2c1</smiles>

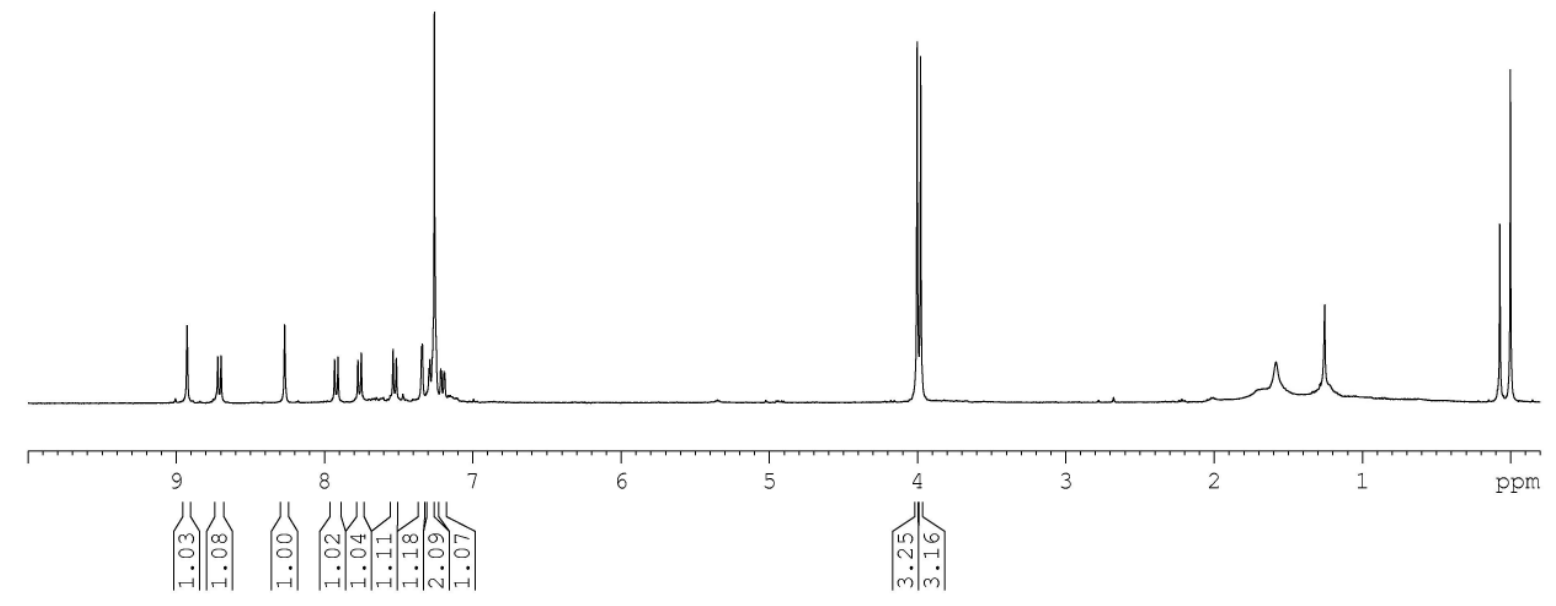

${ }^{13} \mathrm{C}$ NMR (100 MHz) spectra of $\mathbf{2 b}$
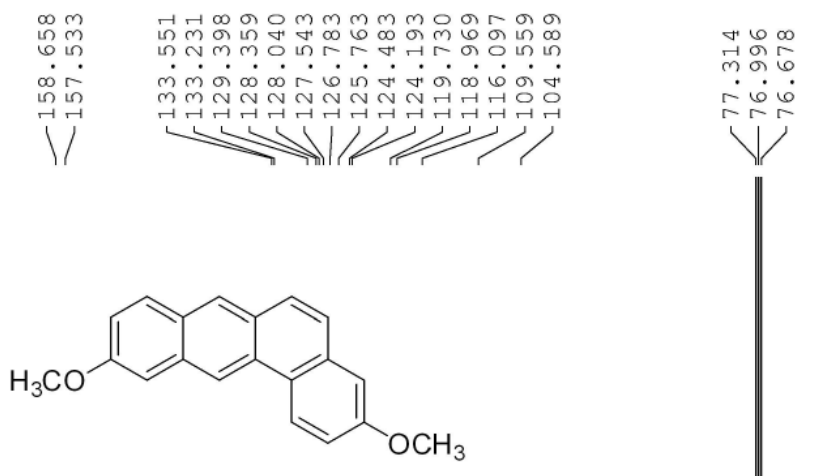

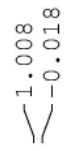

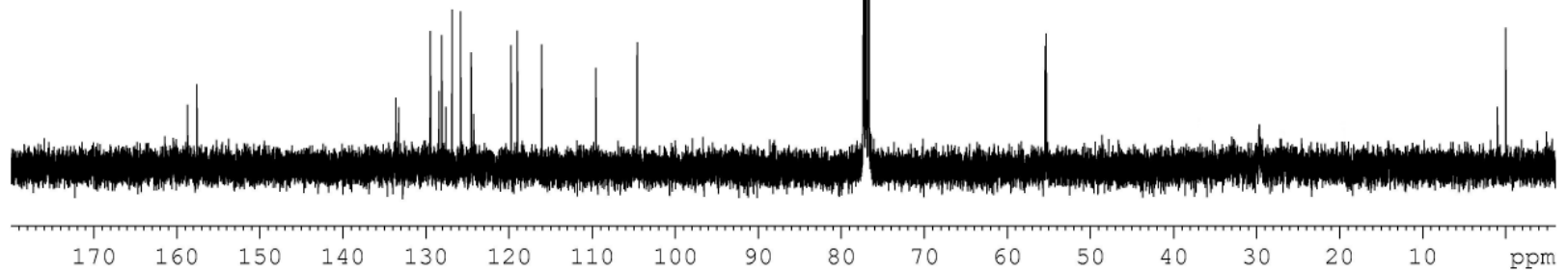


${ }^{1} \mathrm{H}$ NMR (400 MHz) spectra of 2c

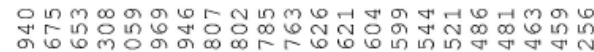

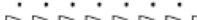

N<smiles>Clc1ccc2cc3ccc4cc(Cl)ccc4c3cc2c1</smiles>
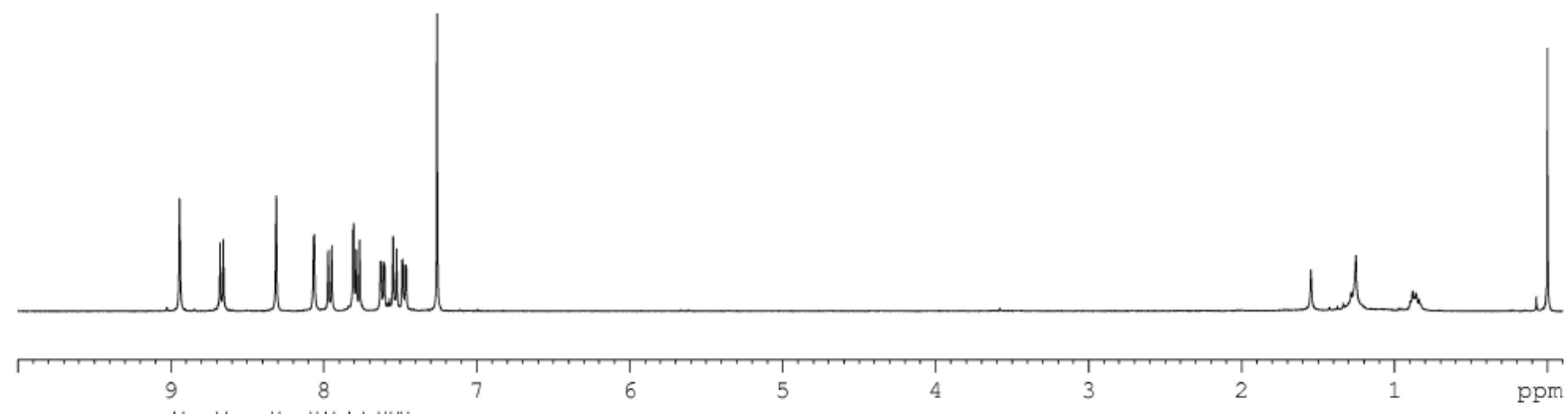

(1)

${ }^{13} \mathrm{C}$ NMR (100 MHz) spectra of 2c

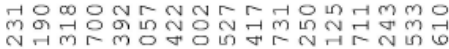

miñ

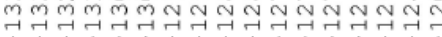

mit।<smiles>Clc1ccc2cc3ccc4cc(Cl)ccc4c3cc2c1</smiles>

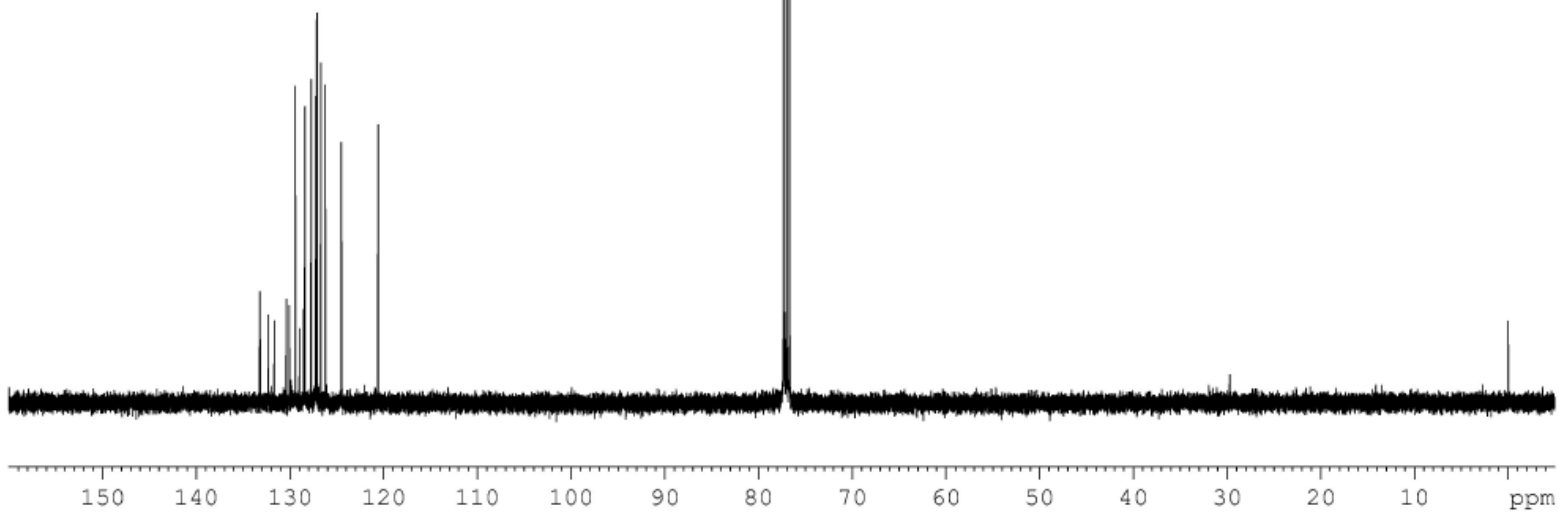


${ }^{1} \mathrm{H}$ NMR (400 MHz) spectra of ad
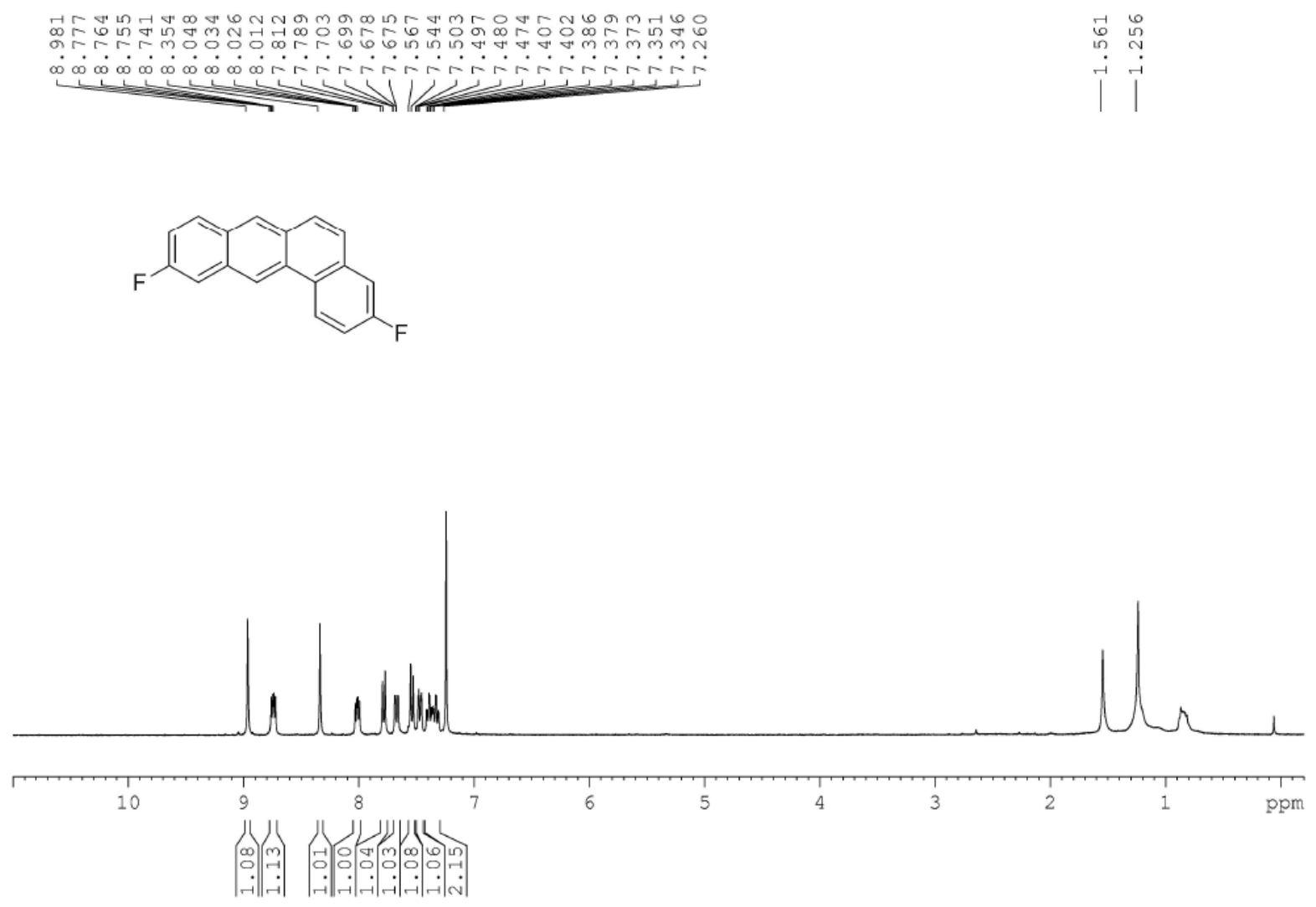

${ }^{13} \mathrm{C}$ NMR (100 MHz) spectra of ad
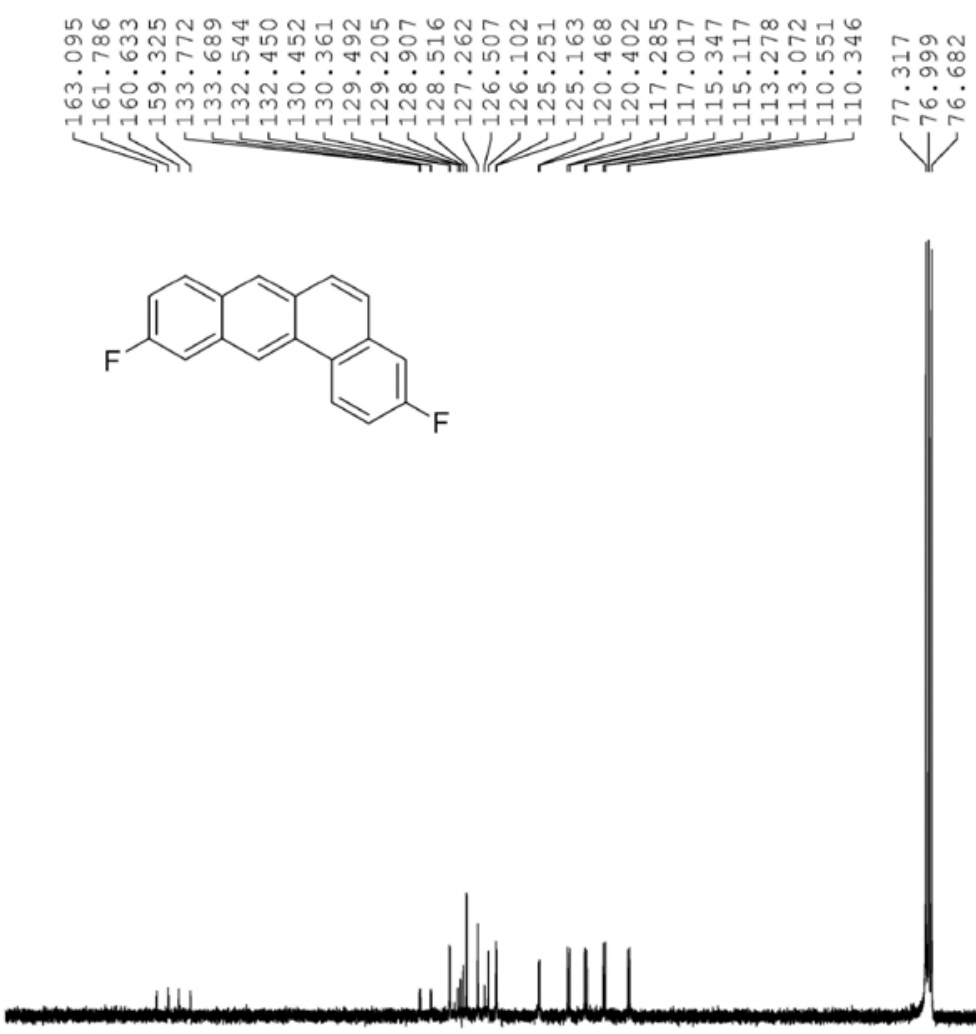

170

160

150

90

80

70

30

20

S12 
${ }^{1} \mathrm{H}$ NMR (400 MHz) spectra of $\mathbf{2 e}$

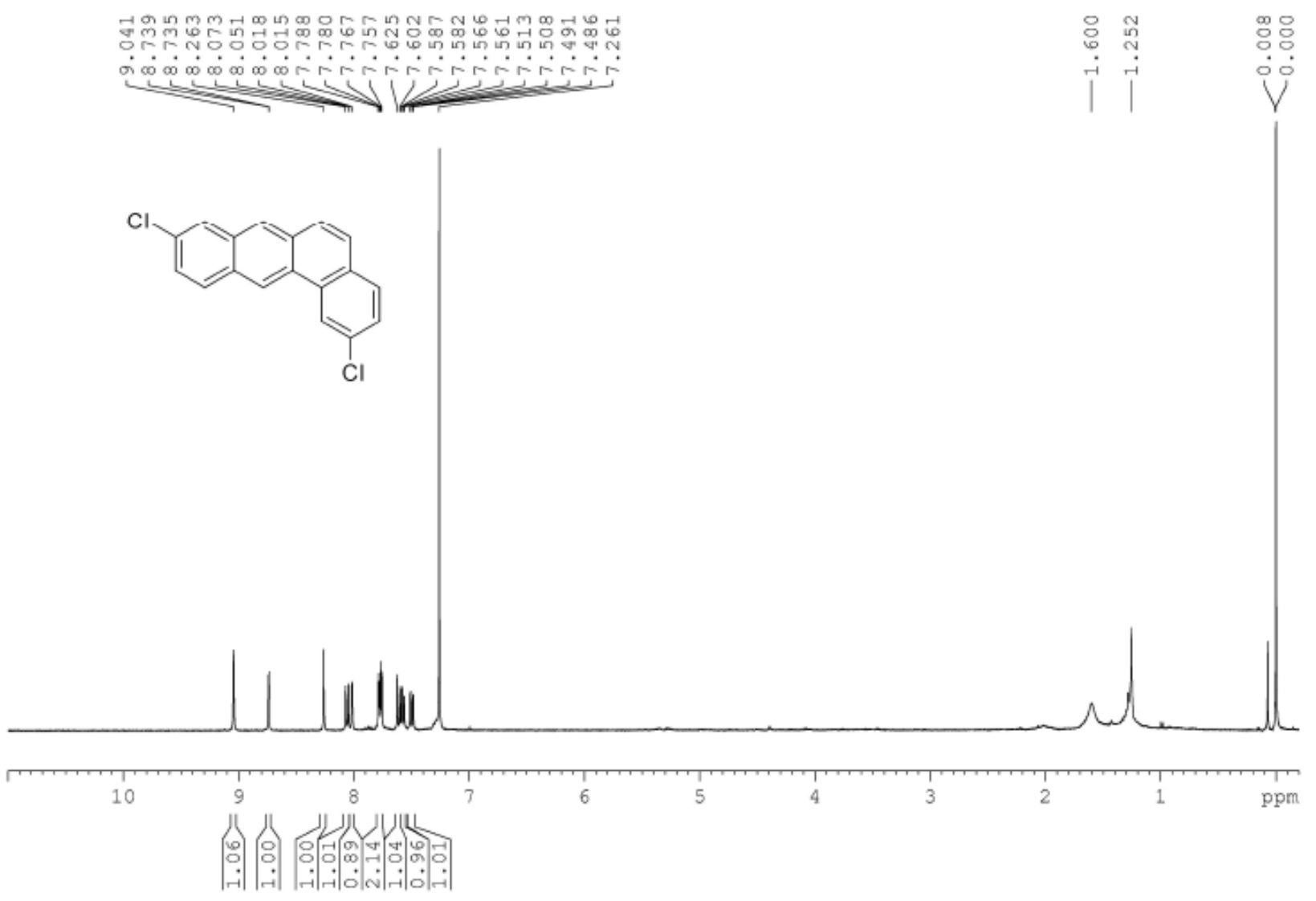

${ }^{13} \mathrm{C}$ NMR (100 MHz) spectra of $\mathbf{2 e}$

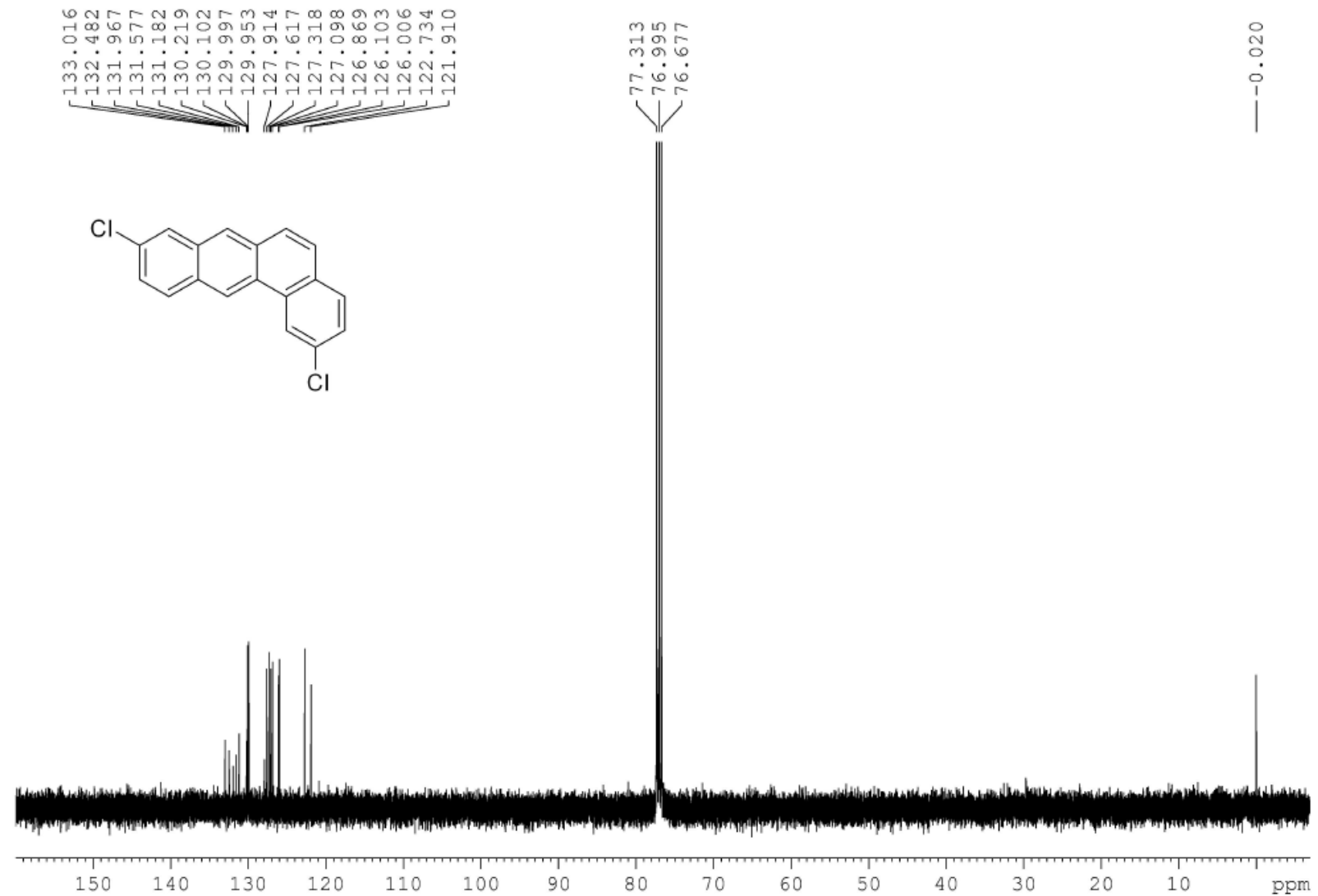


${ }^{1} \mathrm{H}$ NMR (400 MHz) spectra of $\mathbf{2 f}$

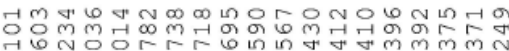

$\cdot \cdot \cdot \cdot \cdot$

$\underbrace{\infty}$

(n)<smiles>Cc1ccc2cc3ccc4ccc(C)cc4c3cc2c1</smiles>

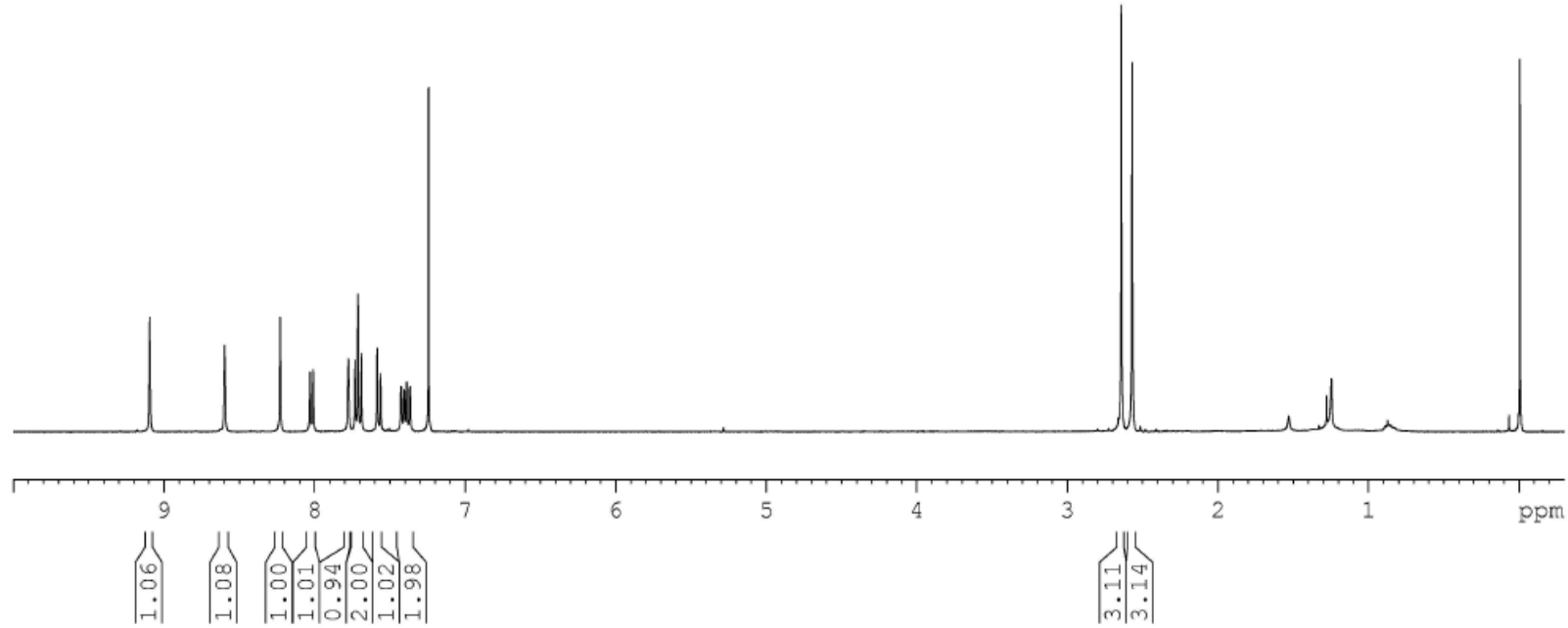

${ }^{13} \mathrm{C}$ NMR (100 MHz) spectra of $\mathbf{2 f}$

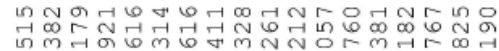
m

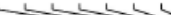<smiles>Cc1ccc2cc3ccc(C)cc3cc2c1</smiles>

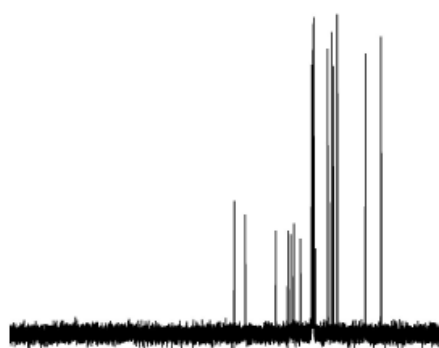


${ }^{1} \mathrm{H}$ NMR (400 MHz) spectra of $\mathbf{2 g}$

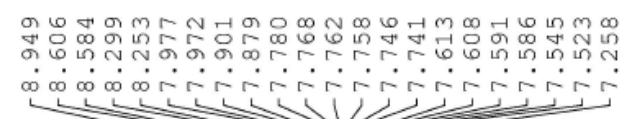



i
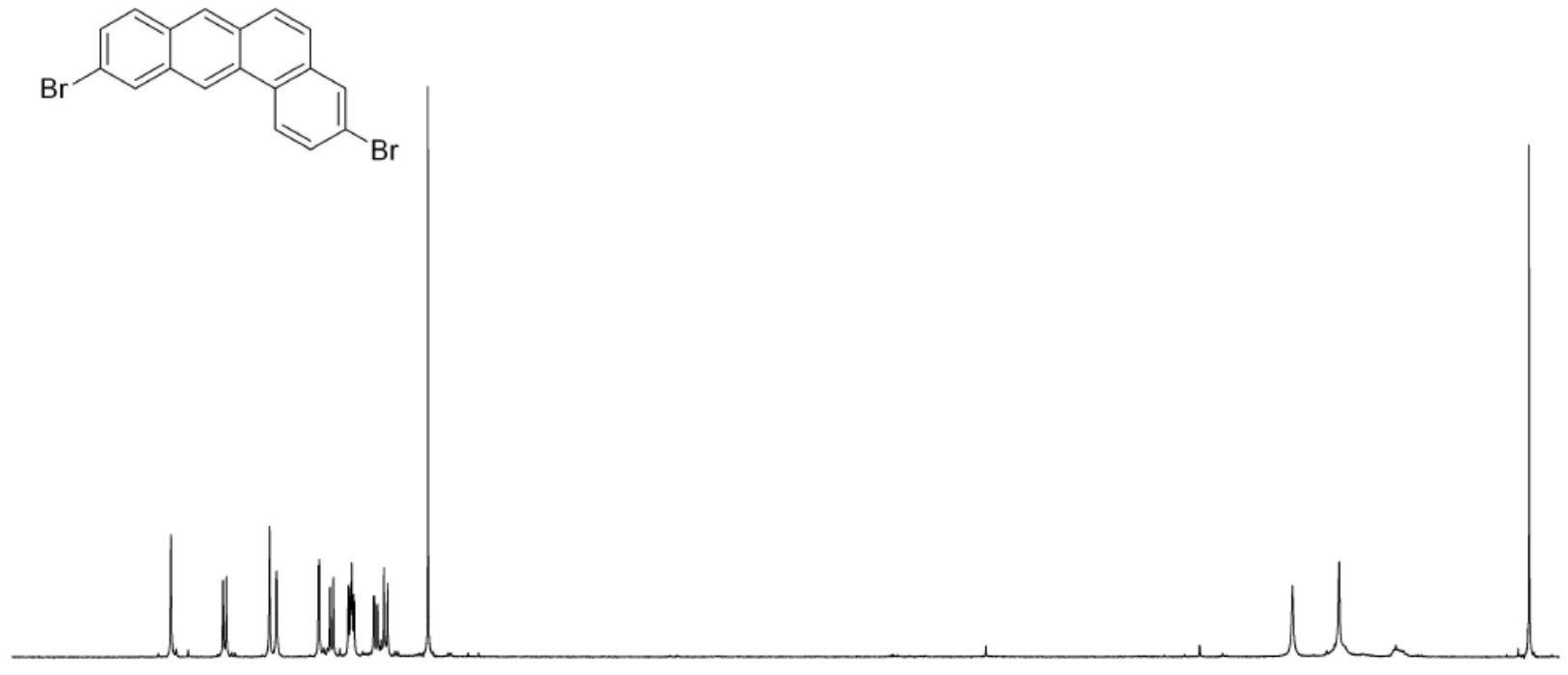

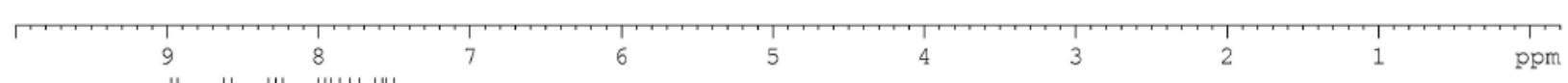

(1)

${ }^{13} \mathrm{C}$ NMR (100 MHz) spectra of $\mathbf{2 g}$
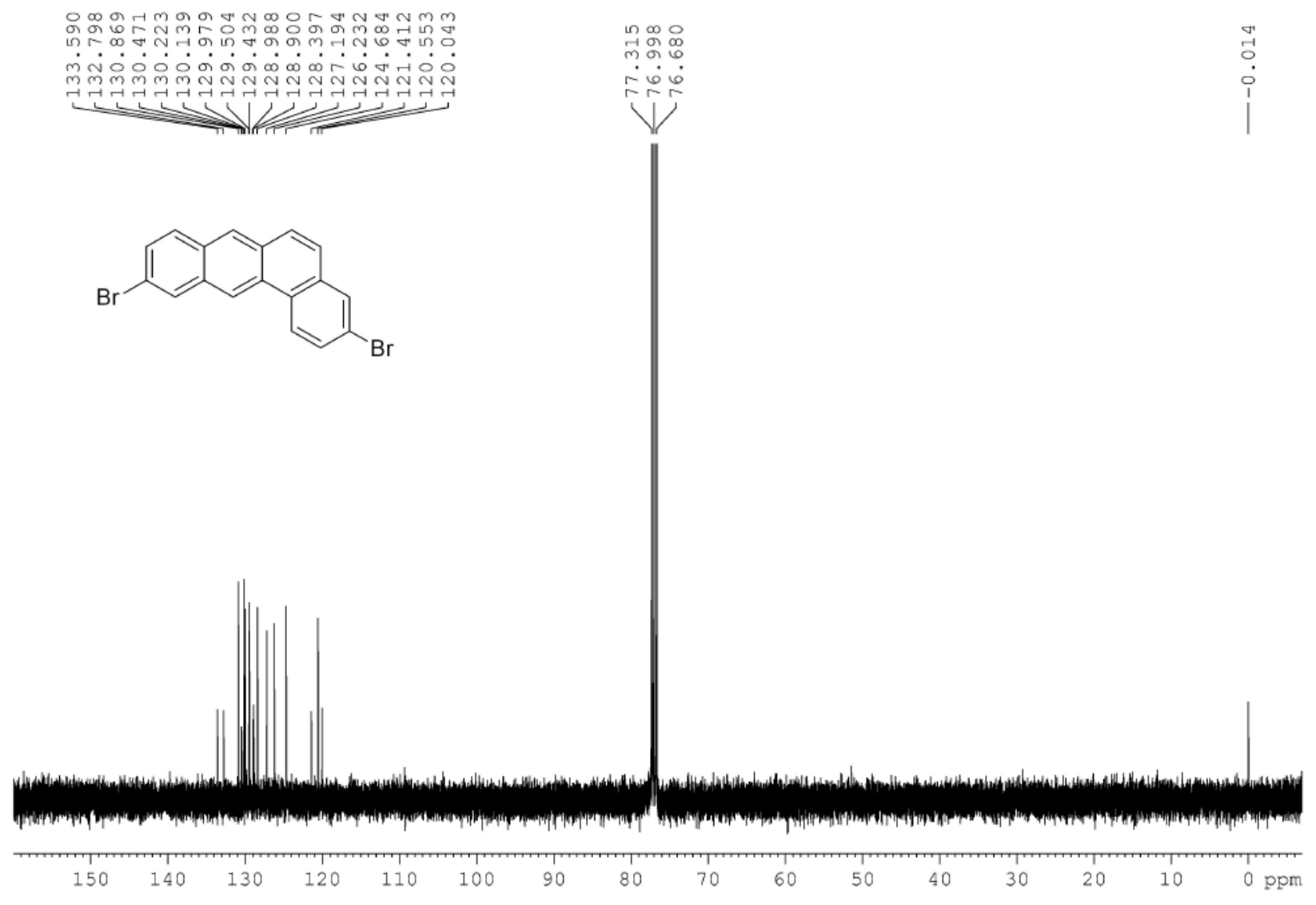

S15 
${ }^{1} \mathrm{H}$ NMR (400 MHz) spectra of $\mathbf{2 h}$

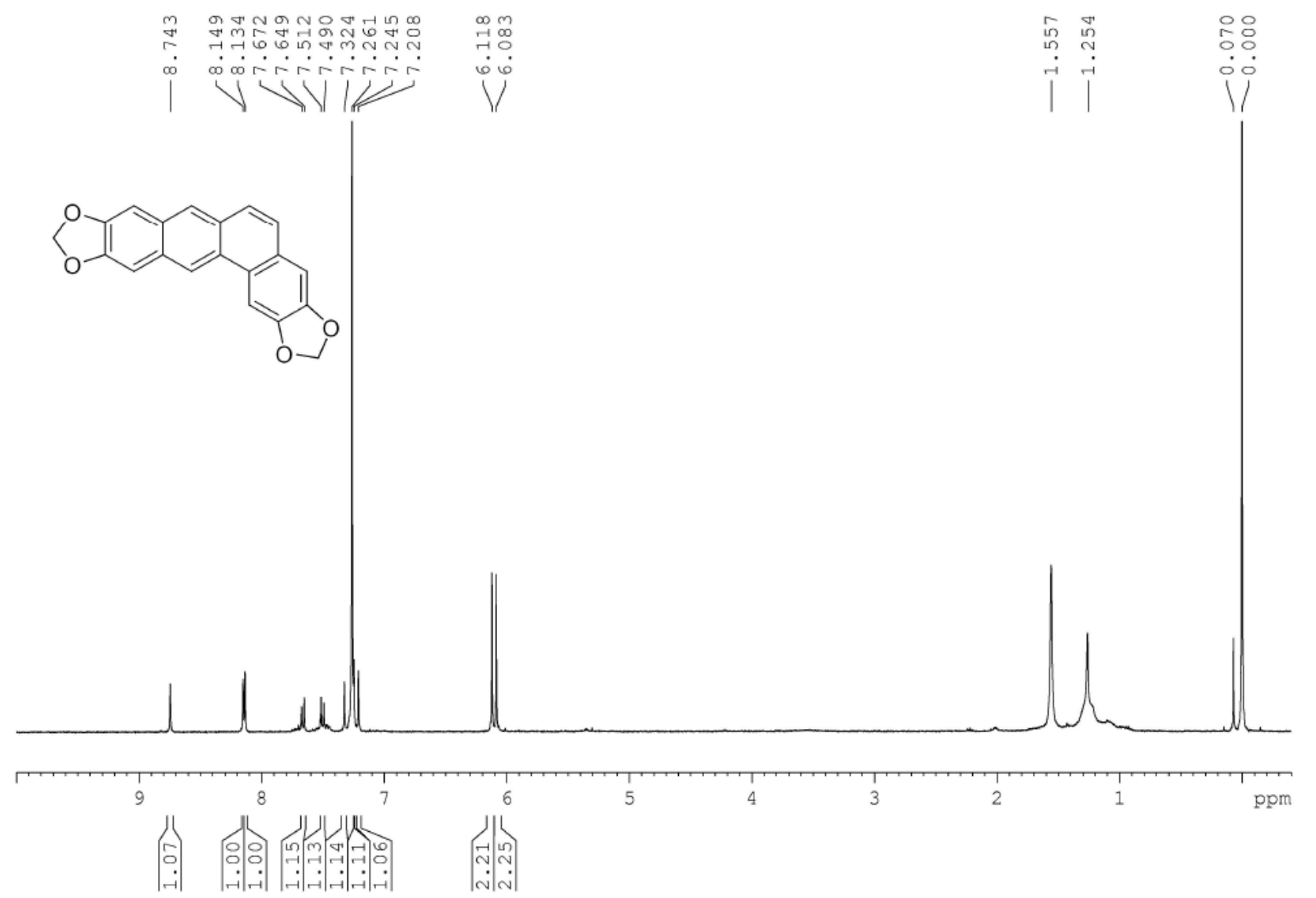

${ }^{13} \mathrm{C}$ NMR (100 MHz) spectra of $\mathbf{2 h}$

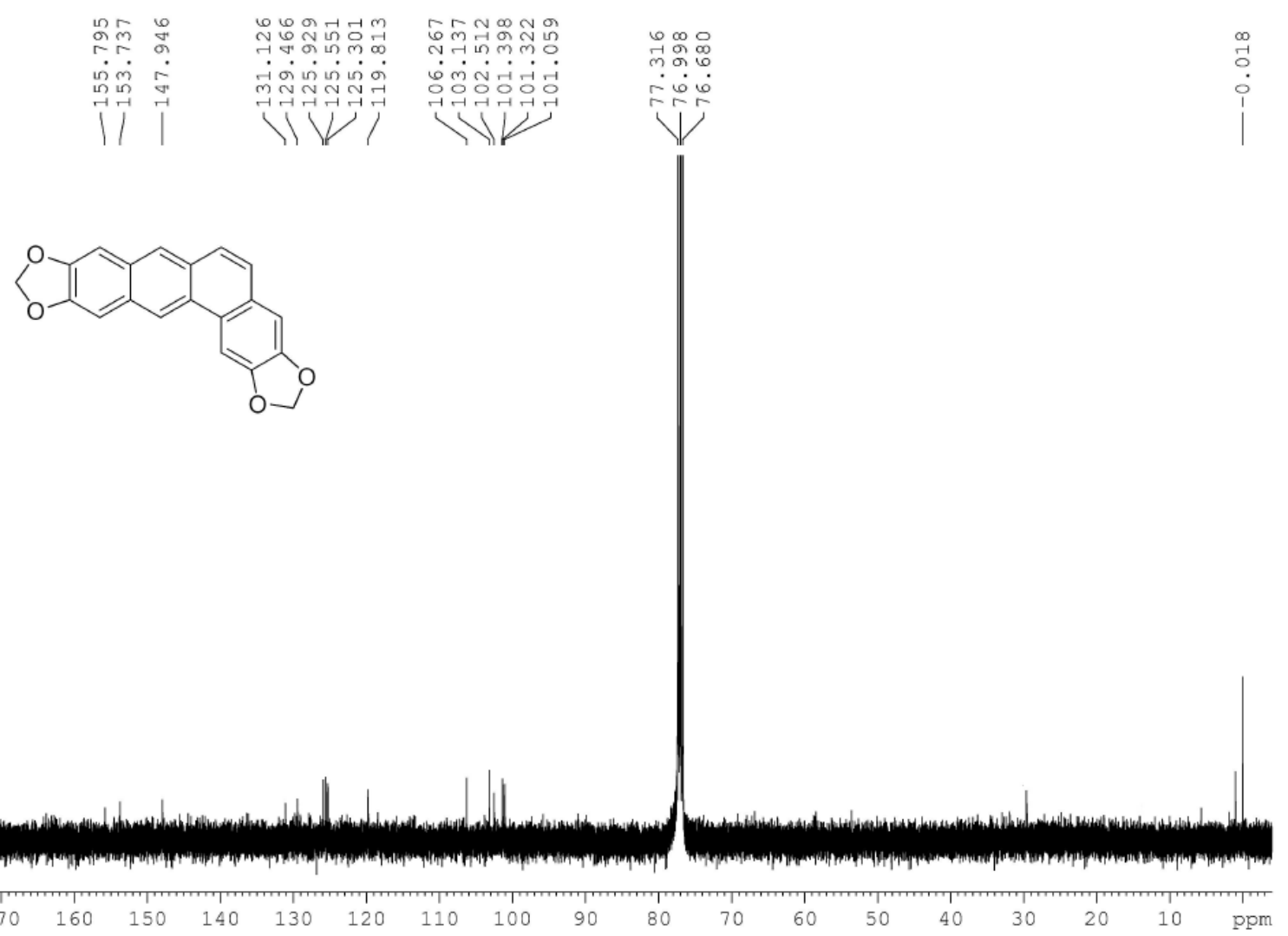


${ }^{1} \mathrm{H}$ NMR (400 MHz) spectra of $2 \mathbf{i}$

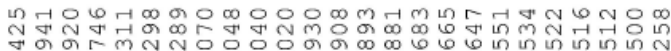

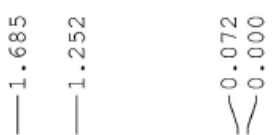
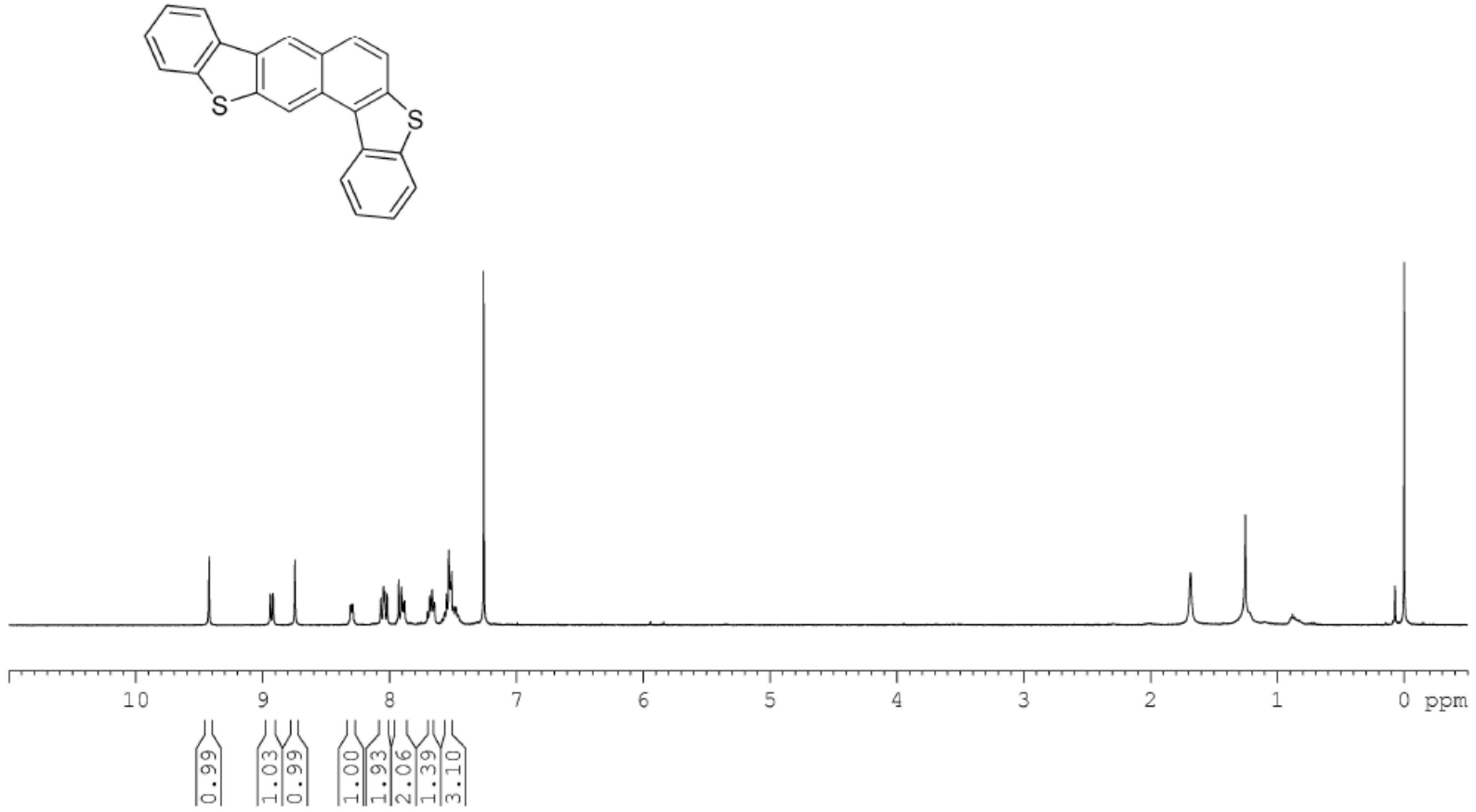

${ }^{13} \mathrm{C}$ NMR (100 MHz) spectra of $\mathbf{2 i}$

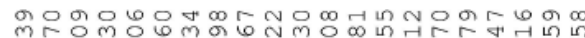

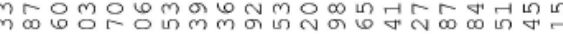

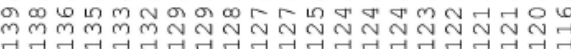

$\rightarrow-1$
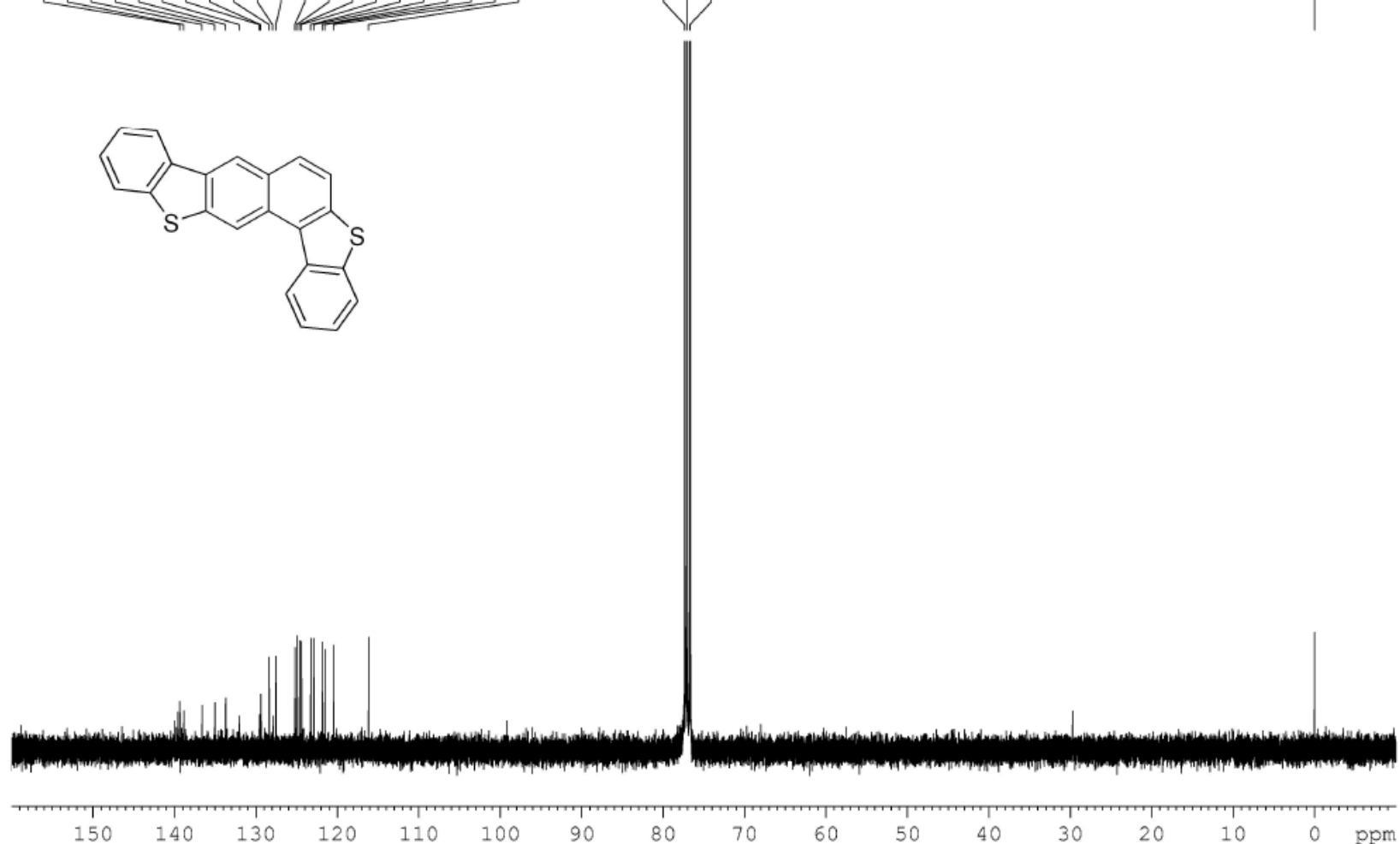
${ }^{1} \mathrm{H}$ NMR (400 MHz) spectra of 3a

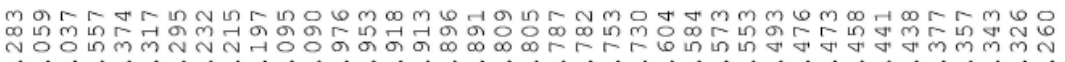

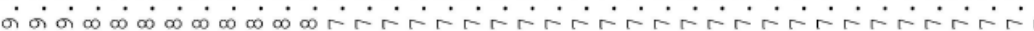

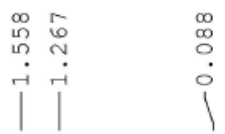<smiles></smiles>

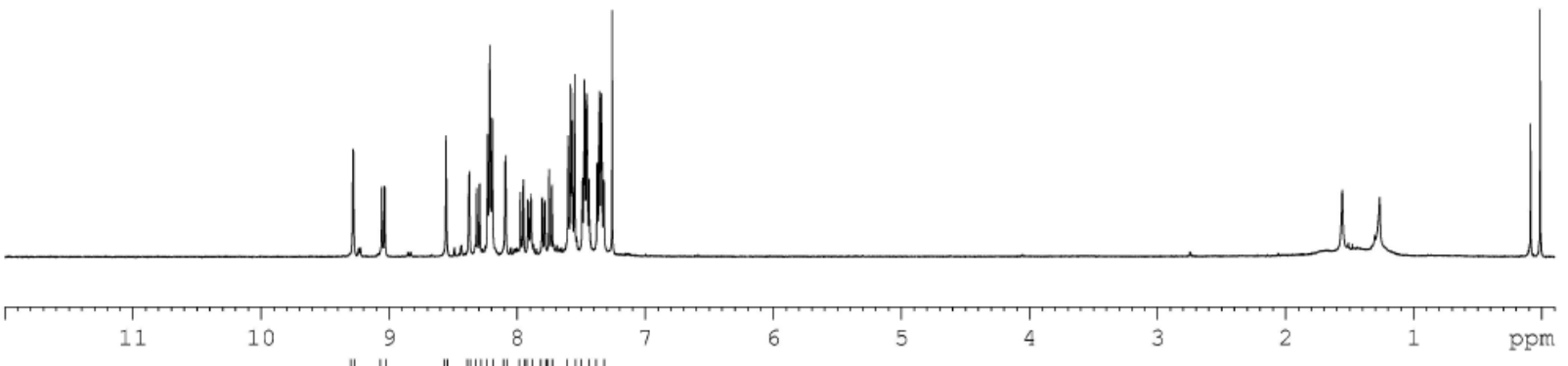

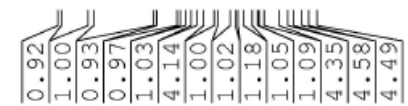

${ }^{13} \mathrm{C}$ NMR (100 MHz) spectra of 3a

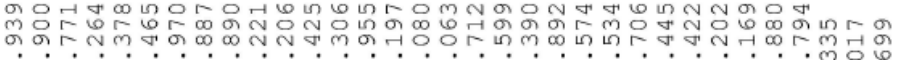

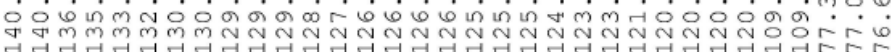

(1)

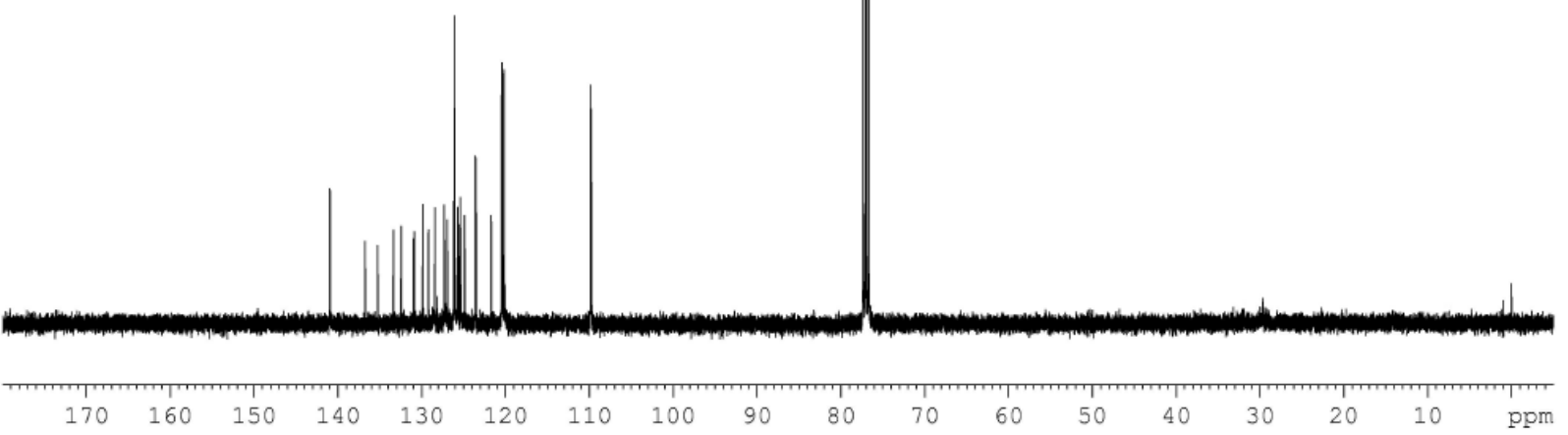


${ }^{1} \mathrm{H}$ NMR (400 MHz) spectra of $\mathbf{3 b}$

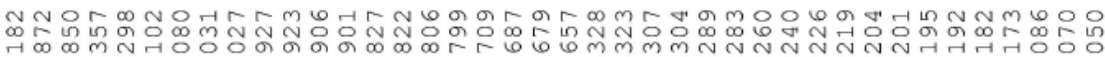

$\dot{\infty} \infty \dot{0} \cdot \dot{0} \cdot \dot{0}$

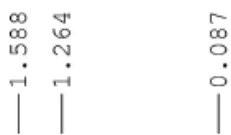<smiles>c1ccc(N(c2ccccc2)c2ccc(-c3ccc4cc5c6cc(-c7ccc(N(c8ccccc8)c8ccccc8)cc7)ccc6cc-5ccc4c3)cc2)cc1</smiles>

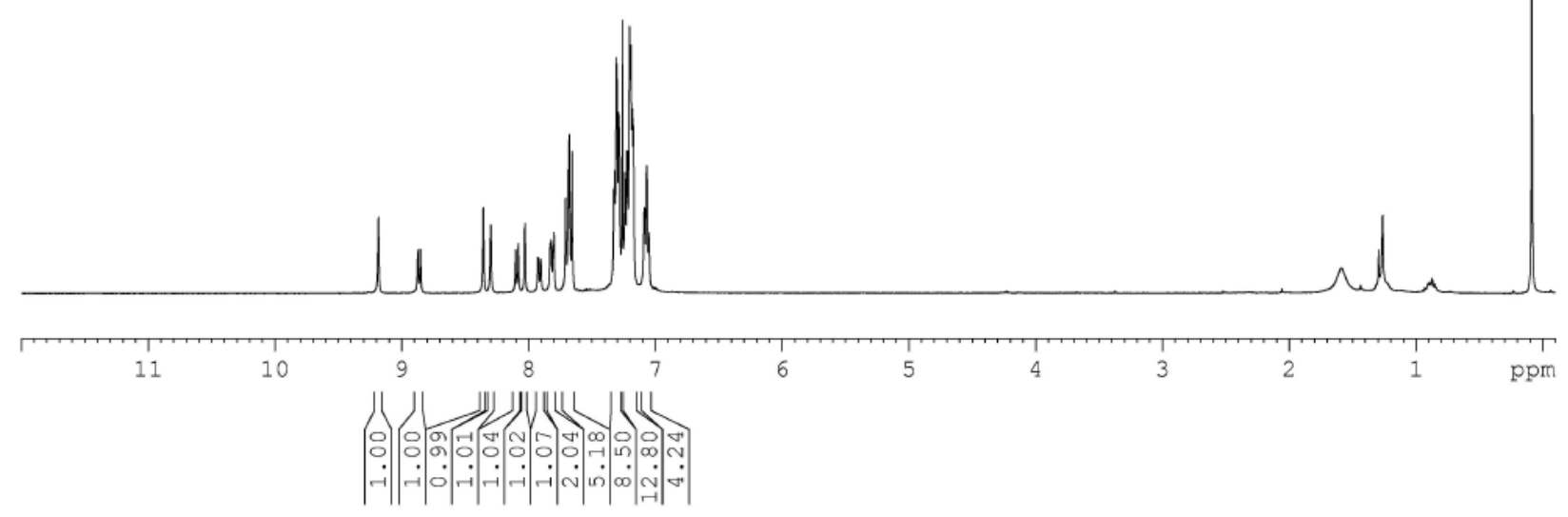

${ }^{13} \mathrm{C}$ NMR (100 MHz) spectra of 3b

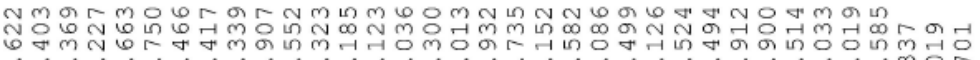

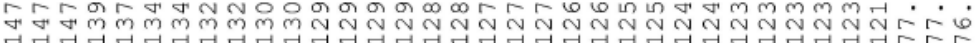

(1)<smiles>c1ccc(N(c2ccccc2)c2ccc(-c3ccc4cc5ccc6cc(-c7ccc(N(c8ccccc8)c8ccccc8)cc7)ccc6c5cc4c3)cc2)cc1</smiles>

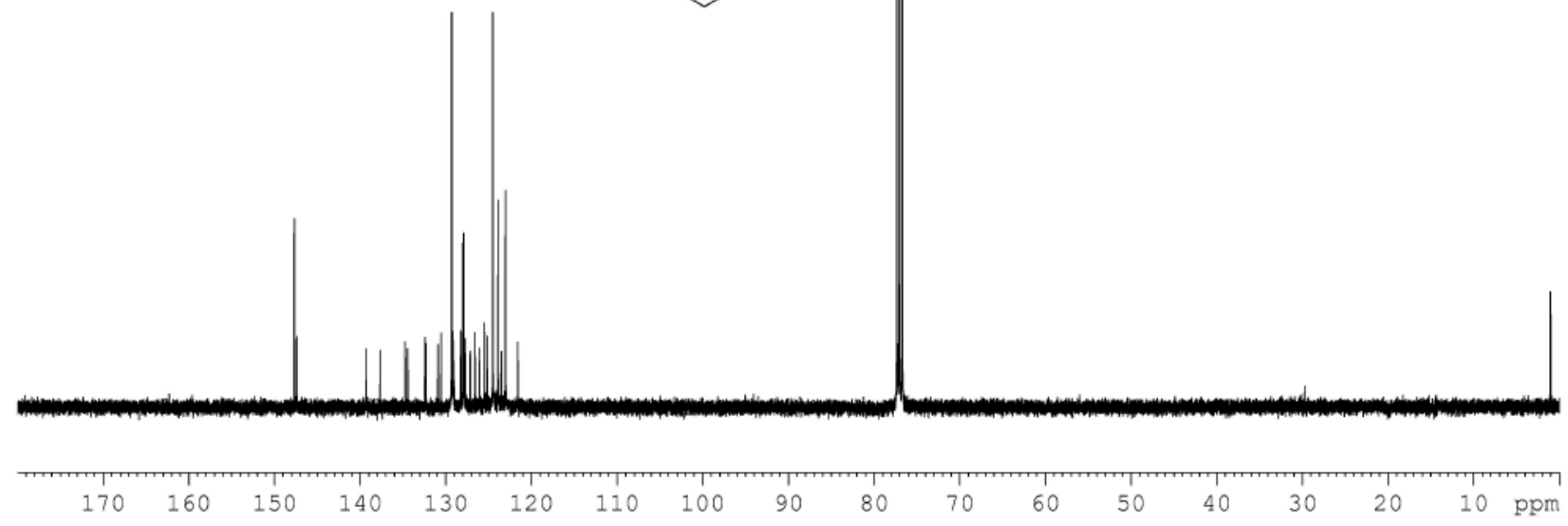

